\title{
QGP hydrodynamical study using energy-momentum in-medium deposition by an extended source.
}

\author{
Julio Cesar Maldonado-Gonzalez ${ }^{1, \star}$, Alejandro Ayala², Isabel Dominguez ${ }^{3}$, and Maria Elena \\ Tejeda-Yeomans ${ }^{1,4}$ \\ ${ }^{1}$ Departamento de Investigación en Física, Universidad de Sonora, Boulevard Luis Encinas J. y Rosales, \\ Colonia Centro, Hermosillo, Sonora 83000, México. \\ ${ }^{2}$ Instituto de Ciencias Nucleares, Universidad Nacional Autónoma de México, Apartado Postal 70-543, Méx- \\ ico Distrito Federal 04510, México. \\ ${ }^{3}$ Facultad de Ciencias Físico-Matemáticas, Universidad Autónoma de Sinaloa, Avenida de las Américas y \\ Boulevard Universitarios, Ciudad Universitaria, C.P. 80000, Culiacán, Sinaloa, México. \\ ${ }^{4}$ Departamento de Física, Universidad de Sonora, Boulevard Luis Encinas J. y Rosales, Colonia Centro, \\ Hermosillo, Sonora 83000, México.
}

\begin{abstract}
The quark-gluon plasma (QGP) is created under extreme conditions, such as the ones prevailing in heavy ion collisions. The characterization of the QGP can be done using high- $p_{T}$ probes such as the partons that are created through hard scatterings in the fireball. These fast-moving partons lose energy and momentum along their traveled path through the medium. The parton deposition of energy-momentum creates an in-medium disturbance that can be described using approximations within relativistic hydrodynamics in a defined regime of the QGP evolution. Based on earlier research in this field, we study the use of extended sources that depend on the location of the parton-jet in the initial stages of the QGP evolution. We explore this approach as a way to complement the current numerical landscape of hydrodynamical QGP studies and to eventually generate initial conditions that can be used as input of hydrodynamical numerical simulations.
\end{abstract}

\section{Introduction}

Current experimental evidence points to the creation of the quark-gluon plasma in heavy ion experiments such as the ones taking place at the Large Hadron Collider (LHC) at CERN and the Relativistic Heavy Ion Collider (RHIC) at BNL. These experiments have allowed a more detailed characterization of the QGP, mainly in the transition phase from quarks to hadrons: they have discovered the QGP to be a strongly-coupled fluid, with low viscosity [1]. In these heavy-ion collisions, hadrons are created and they reach the detectors with large collective velocity. In order to characterize the QGP properties, we can use high $p_{T}$ probes such as hard-scattering partons, that are created in the bulk of the plasma and that travel across it, loosing energy and momentum. In this work we report on the energy-momentum deposition of partons modeled as extended sources and compare with previous results.

^e-mail: julio.maldonadog@gmail.com 


\section{Perturbing the medium in local equilibrium}

In ultra-relativistic heavy-ion collisions the medium never reaches the equilibrium state, but it is possible to consider it in local equilibrium. Local conservation laws guarantee that $\partial_{\mu} \Theta_{0}^{\mu \nu}=0$ and $\Theta_{0}^{\mu \nu}=-P g^{\mu \nu}+(\epsilon+P) u^{\mu} u^{\nu}$, where the medium 4-velocity is $u^{\mu}=(1, \mathbf{u})$ and where the momentum density $\mathbf{g}$ connects with the 3 -velocity as $\mathbf{u}=\mathbf{g} / \epsilon_{0}\left(1+c_{s}^{2}\right)$. $\epsilon_{0}$ is the static background energy density and $c_{s}$ is the sound velocity. Assuming that the disturbance introduced by the parton is small, the medium energy-momentum tensor can be written as $\Theta^{\mu \nu}=\Theta_{0}^{\mu \nu}+\delta \Theta^{\mu \nu}$, where $\Theta_{0}^{\mu \nu}$ is the equilibrium energy-momentum tensor and $\delta \Theta^{\mu v}$ is the perturbation made by the parton [2]. The energy-momentum tensor satisfies $\partial_{\mu} \delta \Theta^{\mu \nu}=J^{\mu}$ and $\partial_{\mu} \Theta_{0}^{\mu \nu}=0$ where $J^{\mu}$ is the source of the disturbance. If we consider a first order approximation in shear $(\eta)$ and ignoring bulk viscosity effects, the perturbation to the energy-momentum tensor will have the following components,

$$
\delta \Theta^{00}=\delta \epsilon, \quad \delta \Theta^{0 i}=\mathbf{g}, \quad \delta \Theta^{i j}=\delta_{i j} c_{s}^{2} \delta \epsilon-\frac{3}{4} \Gamma_{s}\left(\partial^{i} \mathbf{g}^{j}+\partial^{j} \mathbf{g}^{i}-\frac{2}{3} \delta_{i j} \nabla \cdot \mathbf{g}\right)
$$

where the sound attenuation length is $\Gamma_{s} \equiv 4 \eta / 3 \epsilon_{0}\left(1+c_{s}^{2}\right)$ and $\epsilon(t, \mathbf{x})=\epsilon_{0}+\delta \epsilon(t, \mathbf{x})$ with $\epsilon_{0}$ is the energy density of the background fluid, and $\delta \epsilon$ the disturbance energy density. Applying a Fourier Transform to Eq. (1), the longitudinal and transverse components of the momentum density as well as the energy density are given by

$$
\mathbf{g}_{L}=\frac{i\left[\frac{\omega}{k^{2}} \mathbf{k} \cdot \mathbf{J}(\mathbf{k}, \omega)+c_{s}^{2} J^{0}(\mathbf{k}, \omega)\right]}{\omega^{2}-c_{s}^{2} k^{2}+i \Gamma_{s} \omega k^{2}}, \quad \mathbf{g}_{T}=\frac{i \mathbf{J}_{T}}{\omega+i \frac{3}{4} \Gamma_{s} k^{2}}, \quad \delta \epsilon=\frac{i \mathbf{k} \cdot \mathbf{J}(\mathbf{k}, \omega)+J^{0}(\mathbf{k}, \omega)\left(i \omega-\Gamma_{s} k^{2}\right)}{\omega^{2}-c_{s}^{2}+i \Gamma_{s} \omega k^{2}},
$$

where $\mathbf{g}=\mathbf{g}_{L}+\mathbf{g}_{T}$ and $\mathbf{g}_{L}=(\mathbf{g} \cdot \mathbf{k}) \mathbf{k} / k^{2}$.

\section{Localized versus extended source}

The in-medium perturbation that the traveling parton generates can be modeled by a localized source $J^{v}(\mathbf{x}, t)=(d E / d x) v^{v} \delta(\mathbf{x}-\mathbf{v} t)$, as it was done in Ref. [2] where $d E / d x$ is the parton energy-loss per unit length and $v^{v}=(1, \mathbf{v})$ is the parton velocity. In order to model a jet (instead of a parton) passing through the medium, an extended source was proposed in Ref. [3]. Inspired by this proposal, we study a simplified version of an extended source and we make a comparison with the results of Ref. [2] for a localized source. We now consider an extended source

$$
J^{v}(\mathbf{x}, t)=\left(\frac{d E}{d x}\right) v^{v} \frac{1}{(\sqrt{2 \pi} \sigma)^{3}} \exp \left\{-\frac{(\mathbf{x}-\mathbf{v} t)^{2}}{2 \sigma^{2}}\right\}
$$

where the parameter $\sigma$ allows us to control the extension of the energy-momentum deposit. For this study, we will consider $\mathbf{x} \cdot \mathbf{v}=|\mathbf{x}||\mathbf{v}| \cos \theta_{0}$, with fixed $\theta_{0}$. The extended source of Eq. (3) in Fourier space is now,

$$
J^{v}(\mathbf{k}, \omega)=\frac{1}{(2 \pi)^{4}}\left(\frac{d E}{d x}\right) \frac{\sqrt{2 \pi} \sigma}{v \sin ^{3} \theta_{0}} v^{v} \exp \left\{-\frac{2 \sigma^{2}}{v^{2} \sin ^{2} \theta_{0}}\left[\left(1+\frac{1}{4} \sin ^{2} \theta_{0}\right) \omega^{2}-2 \mathbf{v} \cdot \mathbf{k} \omega+v^{2} k^{2}\right]\right\} .
$$

Using Eqs.(2) and (4), we can calculate, for example $\left(\mathbf{g}_{T}\right)_{z}$ as

$$
\left(\mathbf{g}_{T}\right)_{z}=\frac{i}{(2 \pi)^{4}}\left(\frac{d E}{d x}\right) \frac{\sqrt{2 \pi} \sigma}{v \sin ^{3} \theta_{0}} \int \frac{d^{3} k}{(2 \pi)^{3}} \int \frac{d \omega}{2 \pi} \frac{k_{T}^{2} /\left(k_{T}^{2}+k_{z}^{2}\right)}{\omega+i \frac{3}{4} \Gamma_{s}\left(k_{T}^{2}+k_{z}^{2}\right)} \mathrm{e}^{-\frac{2 \sigma^{2}}{v^{2} \sin ^{2} \theta_{0}}\left[\left(1+\frac{1}{4} \sin ^{2} \theta_{0}\right) \omega^{2}-2 \mathbf{v} \cdot \mathbf{k} \omega+v^{2} k^{2}\right]} .
$$



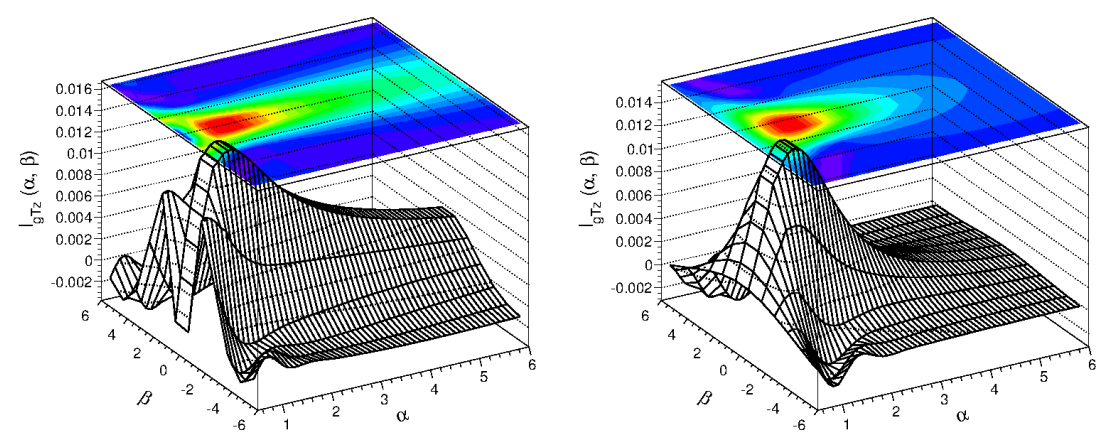

Figure 1: The integrand of $\left(\mathbf{g}_{T}\right)_{z}$ in Eq. (5) is shown on the left hand side for a localized source as was reported in Ref. [2] and, on the right hand side the resulting $\left(\mathbf{g}_{T}\right)_{z}$ with the present analysis of an extended source. We have performed the $\xi$ integral in the $\alpha_{\min } \leq \alpha \leq+6$ and $-6 \leq \beta \leq+6$ range for $\alpha_{\min }=0.5$.

The integrals over $\omega$ and $\mathbf{k}$ can be performed using similar methods as those reported in Ref. [2] where using cylindrical coordinates $\mathbf{k}=\left(k_{T} \cos \phi_{k}, k_{T} \sin \phi_{k}, k_{z}\right)$ and $\mathbf{x}=\left(x_{T} \cos \phi_{x}, x_{T} \sin \phi_{k}, z\right)$, we can describe the momenta both along and transverse to the traveled path, with $\mathbf{v}=v \hat{z}$. Using this and contour integration to calculate $\left(\mathbf{g}_{T}\right)_{z},\left(\mathbf{g}_{T}\right)_{y}$ and $\delta \epsilon$ we arrive at

$$
\begin{aligned}
\left(\mathbf{g}_{T}\right)_{z} & =\frac{1}{(2 \pi)^{\frac{11}{2}}}\left(\frac{d E}{d x}\right) \frac{\sigma}{2 \sin ^{2} \theta_{0}}\left(\frac{2 v}{3 \Gamma_{s}}\right)^{3} \int_{0}^{\infty} d \xi \xi^{2} J_{0}(\beta \xi) \mathrm{e}^{-\alpha \xi} \\
\left(\mathbf{g}_{T}\right)_{y} & =\frac{1}{(2 \pi)^{\frac{11}{2}}}\left(\frac{d E}{d x}\right) \frac{\sigma}{2 \sin ^{3} \theta_{0}}\left(\frac{2 v}{3 \Gamma_{s}}\right)^{3} \int_{0}^{\infty} d \xi \xi^{2} J_{1}(\beta \xi) \mathrm{e}^{-\alpha \xi} \\
\delta \epsilon & =\frac{1}{(2 \pi)^{\frac{11}{2}}}\left(\frac{d E}{d x}\right) \frac{9 \pi \sigma}{4 v \sin ^{3} \theta_{0}}\left(\frac{2 v}{3 \Gamma_{s}}\right)^{3} \int_{0}^{\infty} d \xi\left(A \xi^{2}+B \xi+C\right) J_{0}(\beta \xi) \mathrm{e}^{-\alpha \xi}
\end{aligned}
$$

where $A=-4 \frac{\sigma^{2}}{\sin ^{2} \theta_{0}}\left(\frac{2 v}{3 \Gamma_{s}}\right)\left(\frac{c_{s}}{v}\right)^{2}, B=-1$ and $C=\left(\frac{4 v}{\Gamma_{s}} \frac{\sigma^{2}}{\sin ^{2} \theta_{0}}+v t-\frac{3 \Gamma_{s}}{2 v}-\frac{v \alpha}{\Gamma_{s}}\right)\left(\frac{c_{s}}{v}\right)^{2}$ and where we have used the dimensionless variables $\xi \equiv\left(\frac{3 \Gamma_{s}}{2 v}\right) k_{T}, \alpha \equiv\left(\frac{3 \Gamma_{s}}{2 v}\right)^{-1}|z|$ and $\beta \equiv\left(\frac{3 \Gamma_{s}}{2 v}\right)^{-1} x_{T}$. In order to visualize the energy-momentum deposition, we construct 3D plots of the integrands $\mathrm{I}(\alpha, \beta)$ of Eq. (5), by performing a numerical integration on the dimensionless variable $\xi$. In Figure 1, on the left hand side we show the integrands of $\left(\mathbf{g}_{T}\right)_{z}$ for a localized source as was reported in Ref. [2] and, on the right hand side the resulting integrand of $\left(\mathbf{g}_{T}\right)_{z}$ with the present analysis of an extended source where we have integrated in the $\alpha_{\min } \leq \alpha \leq+6$ and $-6 \leq \beta \leq+6$ range for $\alpha_{\min }=0.5$. Notice how the extended source gives a smother profile near the source, which allows for an easier numerical treatment of the collection of the signal. Figure 2, shows the integrands of $\left(\mathbf{g}_{T}\right)_{y}$ and $\delta \epsilon$ where we have performed the $\xi$ integration in the same range as in the previous figure. On the left column we show the results for a localized source and on the right column the corresponding plots with the present analysis. For $\delta \epsilon$ we use $\Gamma_{s}=0.06, \theta_{0}=\pi / 2$ and $\sigma=0.001$. Again, the signal is smoother and for $\delta \epsilon$ the energy deposit is wider, which is reminiscent of a broad jet. 

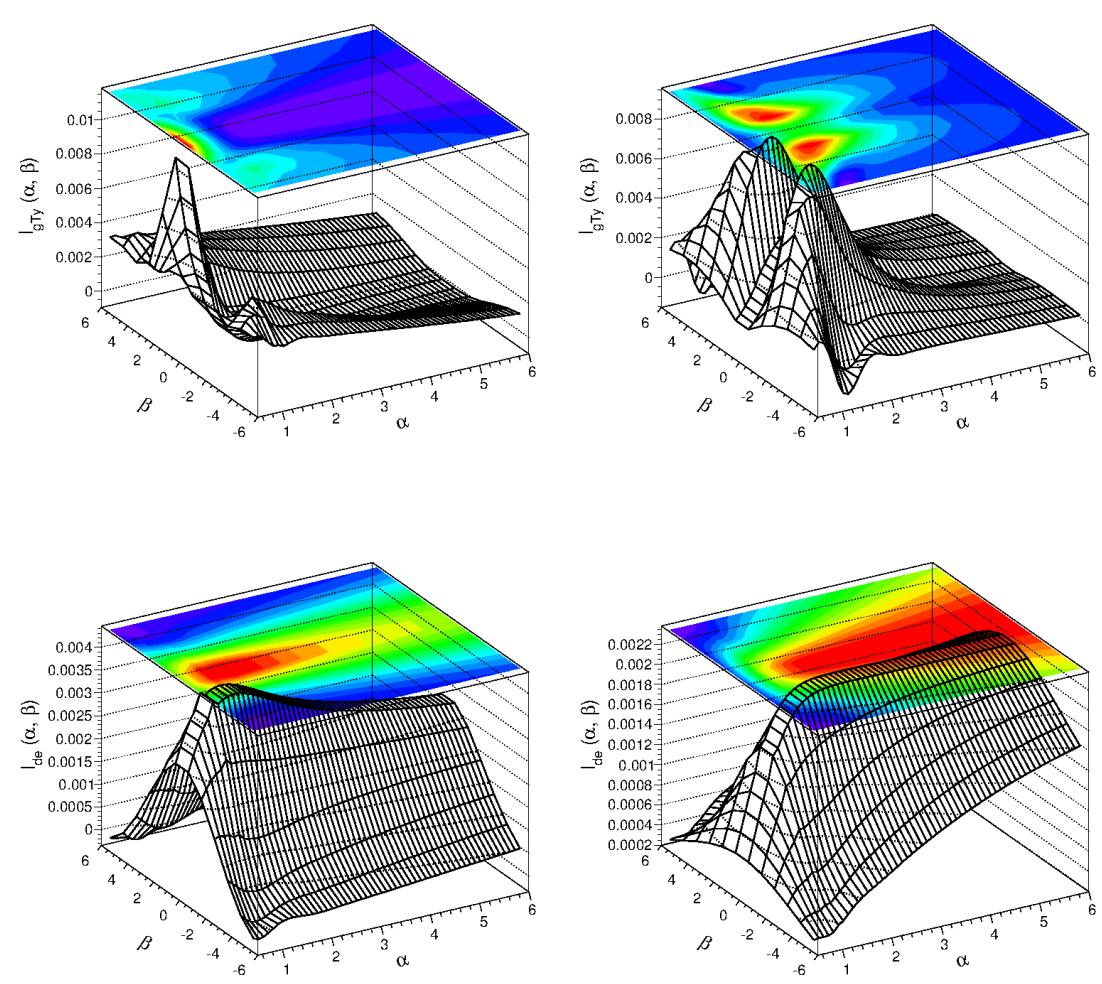

Figure 2: We plot the integrands of $\left(\mathbf{g}_{T}\right)_{y}$ and $\delta \epsilon$ in Eq. (5) where we have performed the $\xi$ integration in the same range as in the previous figure. On the left column we show the results for a localized source as was reported in Ref. [2] and, on the right column the corresponding plots with the present analysis of an extended source. $\left(\mathbf{g}_{T}\right)_{y}$ and $\delta \epsilon$ for a localized source (left) and an extended source (right) with $\alpha_{\min }=0.5$. For $\delta \epsilon$ we use $\Gamma_{s}=0.06, \theta_{0}=\pi / 2$ and $\sigma=0.001$.

In this work we report on the energy-momentum deposition of partons modeled as extended sources and compare with previous results, as a tool to study initial conditions for hydrodynamical numerical simulations and other relevant QGP observables, which will be reported elsewhere [4].

\section{References}

[1] Heavy ions and quark-gluon plasma, home.cern/about/physics/heavy-ions-and-quark-gluonplasma, CERN (2017); L. Cifarelli, L.P. Csernai, and H. Stöcker, The Quark-Gluon Plasma, a nearly perfect fluid, Euro Physics News, DOI:10.1051/epn/2012206.

[2] A. Ayala, I. Dominguez, and M. E. Tejeda-Yeomans, Phys. Rev. C 88, 025203 (2013).

[3] B. Betz, J. Noronha, G. Torrieri, M. Gyulassy, I. Mishustin, and D. H. Rischke, Phys. Rev. C 79, 034902 (2009).

[4] A. Ayala, I. Dominguez, J. C. Maldonado-Gonzalez, M. E. Tejeda-Yeomans, in progress. 